\title{
How does your fat affect your liver when you drink?
}

\author{
Seonghwan Hwang and Bin Gao
}

Laboratory of Liver Diseases, National Institute on Alcohol Abuse and Alcoholism, NIH, Bethesda, Maryland, USA.

\begin{abstract}
White adipose tissue (WAT) dysfunction is generally thought to promote the development of alcoholic liver disease (ALD) in alcoholics by releasing free fatty acids and inflammatory mediators. This explains, at least in part, the synergistic or additive effects of alcohol and obesity on liver disease progression. In this issue of the $J C I$, Shen et al. establish a previously unrecognized concept that brain alcohol sensing enhances thermogenesis of brown adipose tissue (BAT) through sympathetic nerve activation. BAT functions as hepatoprotective machinery to counteract the development of ALD, implying a therapeutic potential of BAT activity modulation for the treatment of ALD.
\end{abstract}

\section{Adipose tissue in alcoholic liver disease}

Alcoholic liver disease (ALD) is a leading cause of chronic liver diseases that ranges from fatty liver to steatohepatitis, cirrhosis, and liver cancer (1). There are no FDAapproved medications for ALD, necessitating the extension of our understanding of its pathogenic mechanisms as well as identification of potent therapeutic targets. Many mechanisms underlying ALD pathogenesis have been identified (1), with recent attention on adipose tissue, which likely plays a more important role in modulating ALD in the modern age than in the past due to the global obesity epidemic (2). Numerous clinical and experimental studies have documented the synergistic or additive effects of alcohol and obesity on liver disease progression well (see review in ref. 2 and references therein). Accumulating data suggest that adipose tissue dysfunction promotes the development of ALD in alcoholics by releasing free fatty acids (FFAs) and inflammatory mediators (2); however, precisely how adipose tissue affects the pathogenesis of ALD still remains largely unknown.

The classical view of adipose tissue as an inert organ for energy storage has been challenged by recent studies providing evidence that adipose tissue is an endocrine organ that actively participates in the regulation of energy metabolism and the immune system by secreting various factors, such as adipokines, cytokines, and chemokines. Accordingly, the dysregulation of adipose tissue biology has been highlighted as a crucial player in the development of inflammatory and metabolic diseases, such as diabetes, nonalcoholic fatty liver disease (NAFLD), and ALD (2, 3). Adipocyte death, which is significantly increased during obesity (4) and alcohol drinking (5), has been recognized as an important initiator for inducing infiltration of macrophages in adipose tissues. Infiltrated macrophages subsequently produce a variety of inflammatory cytokines, such as TNF- $\alpha$ and IL- 6 , and chemokines, such as CCL2, which exacerbate insulin resistance in adipocytes and further recruit immune cells to the inflamed adipocytes $(6,7)$. Adipocyte death has also been shown to induce hepatic fat accumulation and injury, as demonstrated by the observation that reduction of adipocyte death via adipocyte-specific deletion of the Fas gene attenuated fatty liver in high-fat dietfed (HFD-fed) mice (8), whereas induction of adipocyte death via adipocyte-

- Related Article: p. 2305

Conflict of interest: The authors have declared that no conflict of interest exists.

Copyright: () 2019, American Society for Clinical Investigation.

Reference information: J Clin Invest. 2019;129(6):2181-2183. https://doi.org/10.1172/JCI128984. 


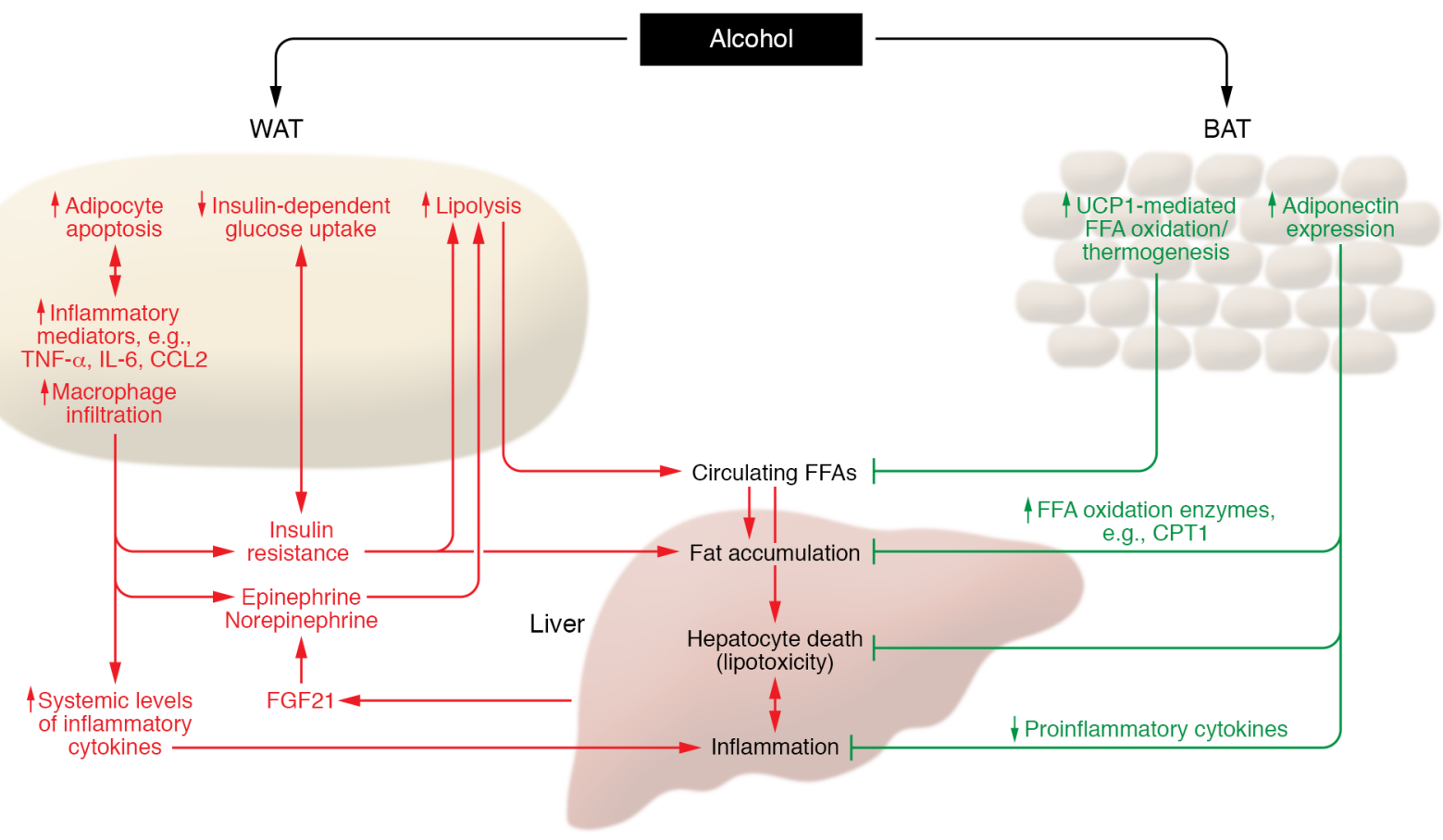

Figure 1. Role of adipose tissue in the pathogenesis of ALD. Excessive alcohol intake induces white adipocyte death and macrophage infiltration, which trigger lipolysis via the elevation of epinephrine and norepinephrine. Alcohol and dysfunctional white adipocytes can also promote lipolysis by induction of insulin resistance and elevation of FGF21. WAT lipolysis subsequently elevates the circulating FFA levels, thereby inducing hepatic FFA influx, lipotoxicity, steatosis, hepatocyte death, and liver inflammation. As opposed to the detrimental effect on WAT, alcohol consumption switches on defensive mechanisms in BAT against ALD development. UCP1-mediated FFA oxidation and thermogenesis are activated by alcohol, which reduces the circulating FFA pool and attenuates FFA-induced hepatocyte death as well as hepatic fat accumulation. Alcohol intake elevates the expression of adiponectin in BAT, which reduces the burden of ALD at multiple levels by suppressing hepatic fat accumulation, hepatocyte death, and liver inflammation. CPT1, carnitine palmitoyltransferase I.

of metabolic syndrome through thermogenesis mediated by mechanisms involving activation of uncoupling protein 1 (UCP1), a protein that is specifically expressed in brown and beige adipocytes. UCP1 uncouples fatty acid oxidation from ATP synthesis in the mitochondria and dissipates energy in the form of heat. Active BAT was originally thought to exist only in infants and rodents, whereas later studies suggest BAT is also present in adult healthy humans, but is less frequently observed in metabolically unhealthy individuals (12-14). The importance of UCP1 and thermogenic activity of BAT in the regulation of energy metabolism was confirmed by the ensuing studies showing that deletion of the $U c p 1$ gene induced obesity at thermoneutrality (15) and that activation of BAT conferred resistance to obesity and metabolic dysfunction in HFD-fed mice $(16,17)$. Additionally, recent studies also suggest a negative correlation between BAT activity and NAFLD development in both humans (18) and rodents (19).

Although BAT has been in the spotlight as a modulator of energy metabolism and potential therapeutic target of NAFLD (20), the association between BAT and ALD has been elusive. Indeed, the influence of alcohol on BAT physiology (e.g., organ mass, thermogenic activity, and lipolysis) has been reported to be inconsistent between studies and thus appears to be contingent on experimental conditions (11). In this issue, Shen et al. extensively investigated the influence of BAT thermogenesis on ALD development by utilizing thermoneutral environment and Ucp1-knockout mice (21). For years, it was not explained why Ucp1 ablation failed to cause obesity in mice, as opposed to the observation that genetic obesity was associated with reduced BAT capacity and activity. This question was solved by an elegant study by Feldmann et al. (15), who performed experiments at thermoneutrality $\left(\right.$ at $\left.30^{\circ} \mathrm{C}\right)$ in which thermal stress was removed, BAT activity was minimized, and thus physiological responses to the alteration in BAT thermogenesis could be better analyzed. This condition is indeed more physiologically relevant, as humans in developed countries do not suffer from metabolic cold stress today (due to clothes and housing) (15). Shen et al. demonstrated that, at thermoneutrality, alcohol feeding significantly elevated the levels of UCP1 expression and mitochondrial respiration complexes, indicating that ethanol intake enhances BAT activity. This notion was further supported by their findings that energy expenditure was elevated and body weight was reduced when mice were fed an alcohol-diet at thermoneutrality. Furthermore, pathological aspects of ALD, such as steatosis, oxidative stress, liver injury, inflammation, and fibrosis, were more prominent in Ucp1-deficient mice exposed to alcohol as compared to the wild-type controls. In contrast, coldinduced BAT activation diminished alcohol-induced steatosis, oxidative stress, and liver injury. These collectively suggest that alcohol-induced activation of UCP1 and thermogenesis in BAT is a defensive mechanism that counteracts the development of ALD. In contrast with the study by Shen et al., the majority of the other stud- 
ies were performed at room temperature, around $22^{\circ} \mathrm{C}$, which is a chronic thermal stress to mice (15). This might explain the discrepancy between the study of Shen et al. and the recent study by Blaner et al. (22) in which alcohol consumption up to four weeks did not increase UCP1 expression.

Intriguingly, the protective role of BAT against the pathogenic mechanisms of ALD does not appear to be mediated by a direct contact of alcohol with brown adipocytes because addition of alcohol to the cultured primary brown adipocytes or differentiated brown adipocytes failed to increase Ucp1 mRNA levels. Instead, as the authors elegantly present, centrally administered alcohol stimulated the brain region (e.g., dorsomedial hypothalamus), which is connected to BAT through sympathetic innervations, and elevated the levels of tyrosine hydroxylase in BAT, a sympathetic nerve marker. Additionally, surgical denervation of BAT reduced the levels of UCP1, tyrosine hydroxylase, and mitochondrial respiration complexes and suppressed then oxygen consumption rate when alcohol was given for four weeks at thermoneutrality, indicating that alcohol stimulation of BAT activity is dependent on BAT innervation.

Systemic insulin resistance stimulates adipose lipolysis and elevates circulating FFA levels, which in turn increases FFA flux into the hepatocytes. This serial process causes lipotoxicity in hepatocytes, which serves as a major pathogenic mechanism of nonalcoholic steatohepatitis. As alcohol consumption also promotes adipose lipolysis in vivo (23), it is plausible that FFA-mediated lipotoxicity significantly contributes to alcohol-induced liver injury. In this regard, the authors' finding that palmitic acid-induced hepatocyte death was attenuated by adiponectin secreted from BAT provides compelling evidence that alcohol induction of BAT activity counteracts the hepatocyte lipotoxicity caused by FFA influx. This notion is also consistent with the previous report showing that adiponectin alleviates ALD in mice through upregulation of fatty acid oxidation genes (e.g., Cpt1) and suppression of proinflammatory cytokine production (24) (Figure 1).

\section{Conclusions}

Collectively, the data from Shen et al. suggest that BAT protects against ALD by activating thermogenesis, producing hepatoprotective adipokines (e.g., adiponectin), and removing circulating FFAs. Several approaches have been under investigation for the pharmacological activation of BAT (e.g., adrenergic receptor agonists, transient receptor potential channel agonists, and thyroid hormones), mostly in the context of the treatment of metabolic syndrome (25). Continuous effort toward the development of BAT activators may also open new avenues for the therapeutic intervention of ALD, beyond the hitherto well-studied obesity and metabolic syndrome.

\section{Acknowledgments}

This work was supported by the intramural program of the National Institute on Alcohol Abuse and Alcoholism, NIH (to BG).

Address correspondence to: Bin Gao, Laboratory of Liver Diseases, NIAAA/NIH, 5625 Fishers Lane, Bethesda, Maryland 20892, USA; Phone: 301.443.3998; Email: bgao@mail.nih.gov.

1. Gao B, Bataller R. Alcoholic liver disease: pathogenesis and new therapeutic targets. Gastroenterology. 2011;141(5):1572-1585.

2. Parker R, Kim SJ, Gao B. Alcohol, adipose tissue and liver disease: mechanistic links and clinical considerations. Nat Rev Gastroenterol Hepatol. 2018;15(1):50-59.

3. Tilg H, Moschen AR, Roden M. NAFLD and diabetes mellitus. Nat Rev Gastroenterol Hepatol. 2017;14(1):32-42

4. Strissel KJ, et al. Adipocyte death, adipose tissue remodeling, and obesity complications. Diabetes. 2007;56(12):2910-2918.

5 . Sebastian BM, et al. Identification of a cytochrome P4502E1/Bid/C1q-dependent axis mediating inflammation in adipose tissue after chronic ethanol feeding to mice. J Biol Chem. 2011;286(41):35989-35997.

6. Weisberg SP, McCann D, Desai M, Rosenbaum M, Leibel RL, Ferrante AW. Obesity is associated with macrophage accumulation in adipose tissue. JClin Invest. 2003;112(12):1796-1808.

7. $\mathrm{Xu} \mathrm{H}$, et al. Chronic inflammation in fat plays a crucial role in the development of obesityrelated insulin resistance. JClin Invest. 2003;112(12):1821-1830.

8. Wueest $\mathrm{S}$, et al. Deletion of Fas in adipocytes relieves adipose tissue inflammation and hepatic manifestations of obesity in mice. J Clin Invest. 2010;120(1):191-202.
9. Feng D, et al. SNAP23 regulates BAX-dependent adipocyte programmed cell death independently of canonical macroautophagy. JClin Invest. 2018;128(9):3941-3956.

10. Kim SJ, et al. Adipocyte death preferentially induces liver injury and inflammation through the activation of chemokine ( $\mathrm{C}-\mathrm{C}$ motif) receptor 2-positive macrophages and lipolysis [published online ahead of print January 25, 2019]. Hepatology. https://doi.org/10.1002/hep.30525.

11. Steiner JL, Lang CH. Alcohol, adipose tissue and lipid dysregulation. Biomolecules. 2017;7(1):E16.

12. van Marken Lichtenbelt WD, et al. Cold-activated brown adipose tissue in healthy men. $N$ Engl J Med. 2009;360(15):1500-1508.

13. Cypess AM, et al. Identification and importance of brown adipose tissue in adult humans. $N E n g l$ JMed. 2009;360(15):1509-1517.

14. Virtanen KA, et al. Functional brown adipose tissue in healthy adults. $N$ Engl J Med. 2009;360(15):1518-1525.

15. Feldmann HM, Golozoubova V, Cannon B, Nedergaard J. UCP1 ablation induces obesity and abolishes diet-induced thermogenesis in mice exempt from thermal stress by living at thermoneutrality. Cell Metab. 2009;9(2):203-209.

16. Seale P, et al. Prdm16 determines the thermogenic program of subcutaneous white adipose tissue in mice. J Clin Invest. 2011;121(1):96-105

17. Bordicchia M, et al. Cardiac natriuretic peptides act via p38 MAPK to induce the brown fat thermogenic program in mouse and human adipocytes. J Clin Invest. 2012;122(3):1022-1036.

18. Yilmaz Y, et al. Association between the presence of brown adipose tissue and non-alcoholic fatty liver disease in adult humans. Aliment Pharmacol Ther. 2011;34(3):318-323.

19. Poekes L, et al. Defective adaptive thermogenesis contributes to metabolic syndrome and liver steatosis in obese mice. Clin Sci. 2017;131(4):285-296.

20. Scheja L, Heeren J. Metabolic interplay between white, beige, brown adipocytes and the liver. J Hepatol. 2016;64(5):1176-1186.

21. Shen H, Jiang L, Lin JD, Omary MB, Rui L. Brown fat activation mitigates alcohol-induced liver steatosis and injury in mice. JClin Invest. 2019;129(6):2305-2317.

22. Blaner WS, et al. Chronic alcohol consumption decreases brown adipose tissue mass and disrupts thermoregulation: a possible role for altered retinoid signaling. Sci Rep. 2017;7:43474

23. Zhao C, et al. FGF21 mediates alcohol-induced adipose tissue lipolysis by activation of systemic release of catecholamine in mice. J Lipid Res. 2015;56(8):1481-1491.

24. Xu A, Wang Y, Keshaw H, Xu LY, Lam KS, Cooper GJ. The fat-derived hormone adiponectin alleviates alcoholic and nonalcoholic fatty liver diseases in mice. J Clin Invest. 2003;112(1):91-100.

25. Betz MJ, Enerbäck S. Targeting thermogenesis in brown fat and muscle to treat obesity and metabolic disease. Nat Rev Endocrinol. 2018;14(2):77-87. 